\title{
ARTICLE
}

$\mathrm{SCl} \&$ Exercise

\section{Feasibility, acceptability, and preliminary efficacy of a handcycling high-intensity interval training program for individuals with spinal cord injury}

\author{
Alicia M. Koontz $\mathbb{1}^{1,2,3} \cdot$ Cecile E. Garfunkel ${ }^{4} \cdot$ Theresa M. Crytzer $\mathbb{C}^{1,2,4} \cdot$ Steven J. Anthony ${ }^{5} \cdot$ Bradley C. Nindl $^{4}$
}

Received: 11 February 2020 / Revised: 28 August 2020 / Accepted: 31 August 2020 / Published online: 9 September 2020

(c) The Author(s), under exclusive licence to International Spinal Cord Society 2020

\begin{abstract}
Study design Pilot nonrandomized clinical trial.

Objectives To examine the feasibility, acceptability, and preliminary efficacy of performing handcycling high-intensity interval training (HIIT) for 6 weeks in wheelchair users with spinal cord injury.

Setting Participant's home.

Methods Participants completed pre- and postgraded exercise stress tests, exercise surveys and 6 weeks of handcycling HIIT. The HIIT program consisted of two weekly, 25 min supervised at-home sessions (2-3 min warm-up, then ten intervals of cycling with a ratio of $1 \mathrm{~min}$ work at $90 \%$ peak power output (PPO) to 1 min recovery at 0-20\% PPO, then 2-3 min cool down). Real-time power output and heart rate were recorded via sensors and a bike computer. The sensor data were analyzed to evaluate training efficacy.

Results Seven of the ten enrolled participants (70\%) completed the study. All but one completed the required 12 sessions. The participants met at least 1 of the HIIT target intensity criteria in 76 out of 89 total sessions (85.4\%) performed. Participants expressed a high level of enjoyment on the Physical Activity Enjoyment Scale, mean (SD) = 114.8 (11.3), and satisfaction with the overall experience. Five of the seven participants (71\%) who completed the study felt an increase in endurance, function, and health. Objective physiological changes showed mixed results.

Conclusions Six weeks of handcycling HIIT appears to be safe, feasible and acceptable. A longer HIIT work interval may be needed to elicit significant physiological responses. Future investigation of the feasibility and efficacy of differing HIIT parameters is needed.
\end{abstract}

Supplementary information The online version of this article (https:// doi.org/10.1038/s41393-020-00548-7) contains supplementary material, which is available to authorized users.

Alicia M. Koontz

akoontz@pitt.edu

1 Department of Veterans Affairs, Rehabilitation Research and Development Service, Human Engineering Research Laboratories, Pittsburgh, PA, USA

2 Department of Rehabilitation Science and Technology, University of Pittsburgh, Pittsburgh, PA, USA

3 Department of Bioengineering, University of Pittsburgh, Pittsburgh, PA, USA

4 Neuromuscular Research Laboratory/Warrior Human Performance Research Center, Department of Sports Medicine and Nutrition, University of Pittsburgh, Pittsburgh, PA, USA

5 Division of Endocrinology and Metabolism, Department of Medicine, University of Pittsburgh, Pittsburgh, PA, USA

\section{Introduction}

Spinal cord injury (SCI) is debilitating to an individual's health and functional capacity and can significantly reduce quality of life. Moreover SCI can contribute to a sedentary lifestyle and a subsequent elevated risk for preventable cardiometabolic and hypokinetic diseases [1]. Physical activity is promoted in persons with $\mathrm{SCI}$ as a means to prevent secondary health conditions. However, a majority of individuals with SCI are inactive [2], have low levels of fitness, and experience numerous barriers to exercise [3].

Current exercise guidelines for SCI include performing a minimum of $40 \mathrm{~min}$ of moderate to vigorous intensity aerobic exercise per week to improve cardiorespiratory fitness and a minimum of 90 min per week for eliciting changes in cardiometabolic health [4]. Not addressed in the guidelines are recommendations for high-intensity interval training (HIIT) which is characterized by relatively brief, 
intermittent bouts of high-intensity (i.e., vigorous) exercise at or near maximum heart rate (HR), separated by periods of low intensity exercise for recovery [5]. Studies involving non-SCI populations have found that performing HIIT exercise enhances the body's ability to produce glucose, lower resting blood pressure, improve $\mathrm{VO}_{2}$ peak and peak power output (PPO), and increase brachial artery flowmediated dilation [6-9].

Little research has been conducted on HIIT in persons with SCI [10]. The feasibility of a home-based self-managed 6 week HIIT program was studied in six manual wheelchair users with SCI [11]. The training was performed three times per week through wheelchair propulsion in the community on various outdoor surfaces, altering between high (30 $\mathrm{s}$ at a rate of perceived exertion (RPE) between 6 and 8 on the BorgCR10 scale) and low (60 s at an RPE between 1 and 2) intensities. The study found that some participants had problems finding safe areas to do HIIT and some participants developed shoulder pain. Intense wheelchair propulsion involves high forces and repetitive motion that can increase the risk of developing pain and an overuse injury $[12,13]$. Therefore performing HIIT from a wheelchair, while practical, may not be the best option for wheelchair users.

Handcycling is a popular and rapidly growing sport and recreational activity among wheelchair users. Handcycling promotes high PPO, and the shoulders sustain lower forces due to the simultaneous pattern of cranking the arms, making it a more efficient form of exercise compared to wheelchair propulsion [14, 15]. Short term (e.g., $\leq 7$ weeks) of handcycling HIIT has been found to improve cardiorespiratory fitness among men and women without disabilities [8, 16]. The goal of this pilot study was to explore the feasibility of a handcycling HIIT training program for wheelchair users with SCI. Feasibility outcomes included acceptability, participant retention, adherence, and preliminary efficacy.

\section{Methods}

\section{Participants}

Recruitment flyers were distributed to local rehabilitation SCI and assistive technology clinics and at local adaptive recreational and sporting events. Flyers and emails were also distributed to potentially eligible participants in a research registry.

The inclusion criteria consisted of (1) SCI that occurred at least 6 months prior to the start of the study, (2) use a manual wheelchair as a primary means of mobility (spending 30+ hours per week), (3) between the ages of 16 and 65 , (4) live within $1 \mathrm{~h}$ of driving distance from the research center, (5) able to transfer independently, (6) adequate strength and upper extremity function to operate a handcycle, and (7) adequate space in a safe location to accommodate the study equipment.

The exclusion criteria were (1) history of fractures or dislocations in the upper extremity from which the participant has not fully recovered, (2) upper limb pain or injury that would interfere with the ability to perform aerobic exercise, (3) no current or recent (last 6 months) participation in a structured fitness program, (4) recent hospitalization for any reason (within the past 3 months), (5) pregnancy, (6) history of coronary artery disease, coronary bypass surgery, or other cardiorespiratory events or conditions, (7) likely to experience clinically significant autonomic dysreflexia and/or orthostatic hypotension in response to performing vigorous exercise, and (8) any other condition deemed a contraindication to participation in vigorous exercise by their primary care physician.

\section{Baseline and postintervention visits}

The following intake data were collected: (1) general demographics; (2) current participation in physically active wheelchair sports or recreational activities (yes/no), and the (3) Physical Activity Scale for individuals with physical disabilities (PASIPD) [17]. The PASIPD is a self-report survey that asks how many days and hours per week are spent participating in light, moderate, and vigorous recreational, work and home physical activities. The total score is expressed in a metabolic equivalent (MET) per hour per day with the maximum possible score being 199.5 MET hour/ day [17].

A symptom-limited exercise stress test was conducted before and after the intervention in an exercise lab using a stationary electronically braked upper extremity ergometer (Lode B.V., Groningen, The Netherlands). Participants were fitted with a latex free face mask and a Garmin HR monitor (Garmin International Inc., Olathe, KS). The mask was connected to a Moxus metabolic cart (AEI Technologies, Inc., Bastrop, TX). The test was conducted following American College of Sports Medicine guidelines with work rate incrementally increasing each minute [18]. Participants were verbally and visually cued to maintain a 55-65 revolution per minute (RPM) throughout the test. The resistance started initially at $5 \mathrm{~W}$ and was increased by $10 \mathrm{~W}$ every stage or minute until the participants reached volitional exhaustion or dropped below 55 RPM cadence for $20 \mathrm{~s}$. Oxygen consumption $\left(\mathrm{VO}_{2}\right)$ was recorded every $20 \mathrm{~s}$. A respiratory exchange ratio greater than 1.1 and Borg's RPE $\geq 15$ was used to determine if a maximum $\mathrm{VO}_{2}$ was reached.

\section{Intervention}

The HIIT training sessions were conducted in the participant's home with a study trainer. Participants could 

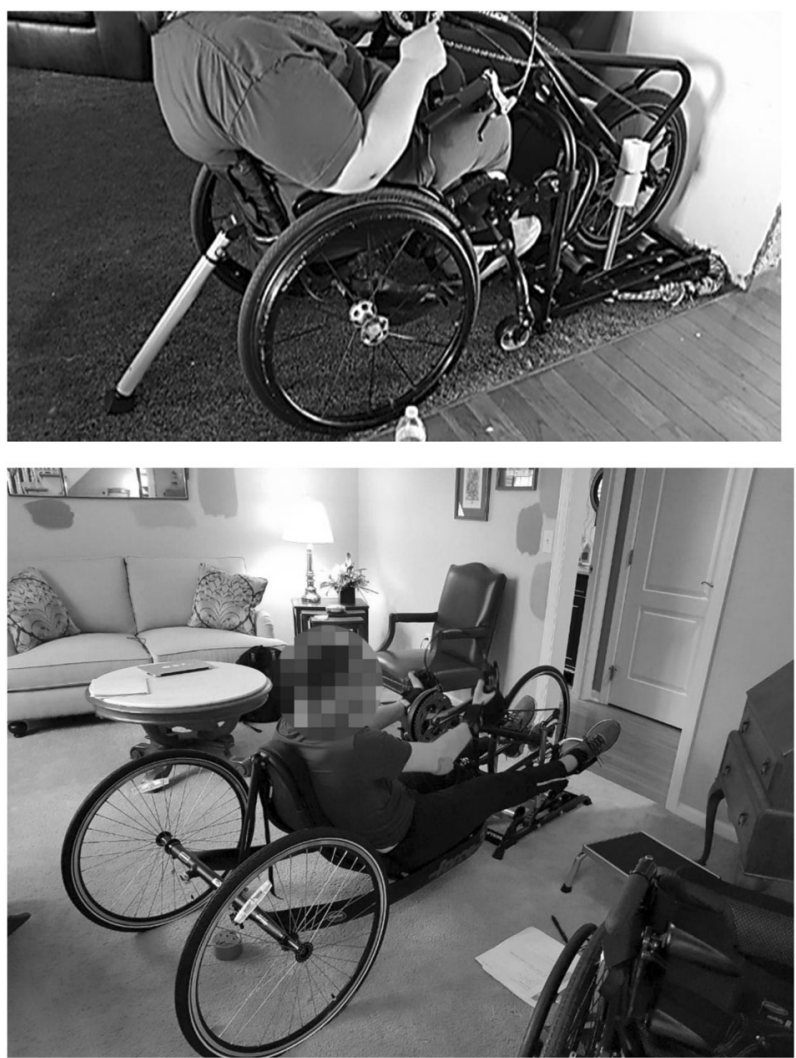

Fig. 1 Example HIIT training setups used in the study. The top figure shows the add-on handbike setup. The bottom figure shows the full handcycle setup.

choose to train with (1) a study handcycle (Top End Force 3, Invacare, Elyria, $\mathrm{OH}$ ), (2) their own handcycle if they owned one, or (3) an add-on Quickie Attitude handbike (Fig. 1) (Sunrise Medical, Fresno, CA). Two standard 17" handcycle wheels and the front wheel of the add-on were custom laced with a power sensing hub (PowerTap, SRAM, LLC, Spearfish, SD). The Powertap hub has a high level of accuracy and reliability $[19,20]$. If the participant chose setup \#2, one of the instrumented wheels was installed onto their handcycle for the training. A cadence sensor (Garmin) was mounted to the crank handle of each bike (Fig. 2). A HR sensor (Garmin) was attached around the participant's chest at the level of the xyphoid process via a strap. All sensors were connected via Bluetooth to a bike computer (Garmin Edge 520) which collected the sensor data during each session (Fig. 2). The bike computer was programmed to display training targets (e.g., power outputs) and the elapsed timing of the work and recovery phases to the user. The front tire of the participants handcycles or add-on bike were positioned on a stationary roller system designed specifically for indoor handcycle training (Overdrive Handcycle Trainer with Rhythm accessory, SportsCrafters, Granger, IN).
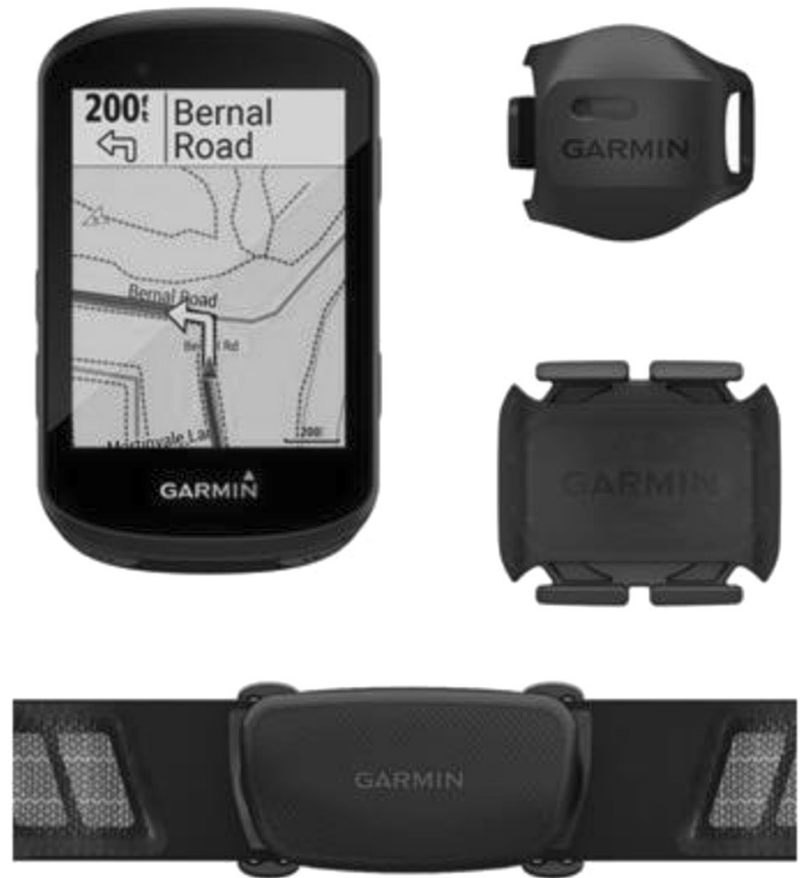

Fig. 2 The Garmin 520 bike computer and sensors used in the study.

The parameters for HIIT were selected based on high tolerance and effectiveness among sedentary, inactive, and clinical populations [5]. The 90\% PPO was determined based on the baseline exercise stress test. The handcycle HIIT programming consisted of 2 days per week of $25 \mathrm{~min}$ of supervised at-home sessions. Each home session began with a 2-3 min warm-up on the handcycle. The warm-up was followed by ten intervals of handcycling HIIT with a ratio of 1 min work at $90 \%$ PPO to $1 \mathrm{~min}$ of recovery at $0-20 \%$ PPO. A 2-3 min cool down was completed at the end. Participants were encouraged, but not required, to perform additional unsupervised HIIT workouts on their own and were shown how to use the equipment to record the sensor data for these workouts. The computer screen was programmed to display the real-time power that was generated during exercise along with a target power range $(90 \% \mathrm{PPO} \pm 5 \mathrm{~W}$ during the work phases). Participants' RPEs and visual exercise cues were used to adjust the power targets if necessary. For example, if the participant reported a RPE that was greater than 15 for the first exercise interval [21] and/or they were having difficulty maintaining the targeted power over the course of the work phases, the target range was reduced by $1-15 \mathrm{~W}$. If the participant reported that it was too easy $(\mathrm{RPE}<13$, somewhat hard) after the first interval and the trainer could visually see that the participant was not working very hard, the power range was increased until the desired effects (e.g., difficulty talking, near breathlessness state, etc.) were seen. Training data from the bike computer were uploaded to the Garmin Connect application for storage and further analysis. 


\section{Measurements}

\section{Adherence to HIIT sessions and intensities}

The number of supervised and unsupervised sessions for each participant was determined. Adherence was calculated based on the total number of supervised sessions that were completed divided by the total number of supervised sessions that were required during the 6-week time period (e.g., 12 sessions completed $=100 \%$ adherence). Session intensity adherence was calculated as a percentage of the number of sessions that met each of the below criteria individually and collectively divided by the total number of sessions that the participant completed. During data analysis it was discovered that some of the HIIT sessions elicited higher HRs than those found during the exercise tests implying that maximal HR was not achieved for some participants. It is possible that peripheral fatigue or other factors (e.g., medication) may have limited or altered the HR responses. For this reason, the highest HR attained during either the pre- or postexercise test or any HIIT session (HRMax) was identified and used to gauge if participants had met the intensity criteria for the HR metric:

(1) Average peak HR during each interval (averaged over all intervals in the session) [21] was at least $90 \%$ of the peak HRMax.

(2) Average work interval power (averaged over all intervals for the session) was within or higher than the targeted power range.

(3) RPE as 17 "very hard" or higher during the last three work intervals of the session $[10,21]$.

\section{Acceptability measures}

The Physical Activity Enjoyment Scale (PACES) [22] is an 18 item scale that is used to measure how individuals feel about the physical activity they are doing. This was administered one time at the end of the intervention period. Each item is measured using a seven-point bipolar rating scale. Higher PACES scores reflect greater levels of enjoyment. A study-specific survey was also administered at the end of the study to collect overall satisfaction with the exercise program and perceived effectiveness of the training.

\section{Fitness measures}

PPO was the highest workload participants could maintain for at least $20 \mathrm{~s}$. HR was measured continuously and sampled at $1 \mathrm{~Hz}$. The highest value obtained during the test was taken as the peak HR. Oxygen consumption $(\mathrm{ml} / \mathrm{min}$ and $\mathrm{ml} / \mathrm{kg} / \mathrm{min}$ ) was continuously measured and averaged over
$20 \mathrm{~s}$ intervals. The highest $20-\mathrm{s}_{2}$ value obtained during the test was used as the peak $\mathrm{VO}_{2}$ [23]. In addition, the length of the test starting at the beginning of the first work stage until the point of termination was determined.

\section{Statistical analyses}

Descriptive statistics (counts, percentages, means, standard deviations) were determined for the study measures. A paired $t$-test was used to evaluate changes in the fitness measures (SPSS v.25, IBM). The level of significance was set at 0.05 .

\section{Results}

\section{Feasibility of recruitment and retention}

Fifteen individuals expressed interest in the study and 14 screened eligible by telephone. Four were lost to enrollment for various reasons, leaving ten who enrolled in the study (Table 1). One participant (S6) after finishing six training sessions developed a pressure ulcer unrelated to study procedures and withdrew (Table 2). Another participant (S8) dropped after the baseline testing because he started a new job. Participant S10 experienced a fall outside of the study and broke her femur during the first week of training.

\section{Adherence to HIIT sessions and intensities}

Six of the seven participants who completed the study were $100 \%$ compliant with meeting the minimum number of supervised sessions (Table 2). One participant (S4) only performed six supervised sessions (50\% adherence rate) due to personal and work-related scheduling conflicts. One participant (S3) did one extra supervised session asked to make up for a session where he was having bad spasms. Another participant (S2) did three additional sessions on his own.

The participants met at least 1 of the HIIT target intensity criteria for 76 out of 89 total sessions (85.4\%) performed in the study (Table 2). The criteria most often met was power (72\%) followed by RPE (53\%) and HR $(46 \%)$. Around $60 \%$ of the sessions met at least two of the criteria and $24 \%$ met all three criteria. Detailed training results for each participant can be found in the Supplemental materials.

\section{Acceptability measures}

The participants enjoyed HIIT to a high degree (Table 3). The lowest scored item was "I find it energizing" mean 


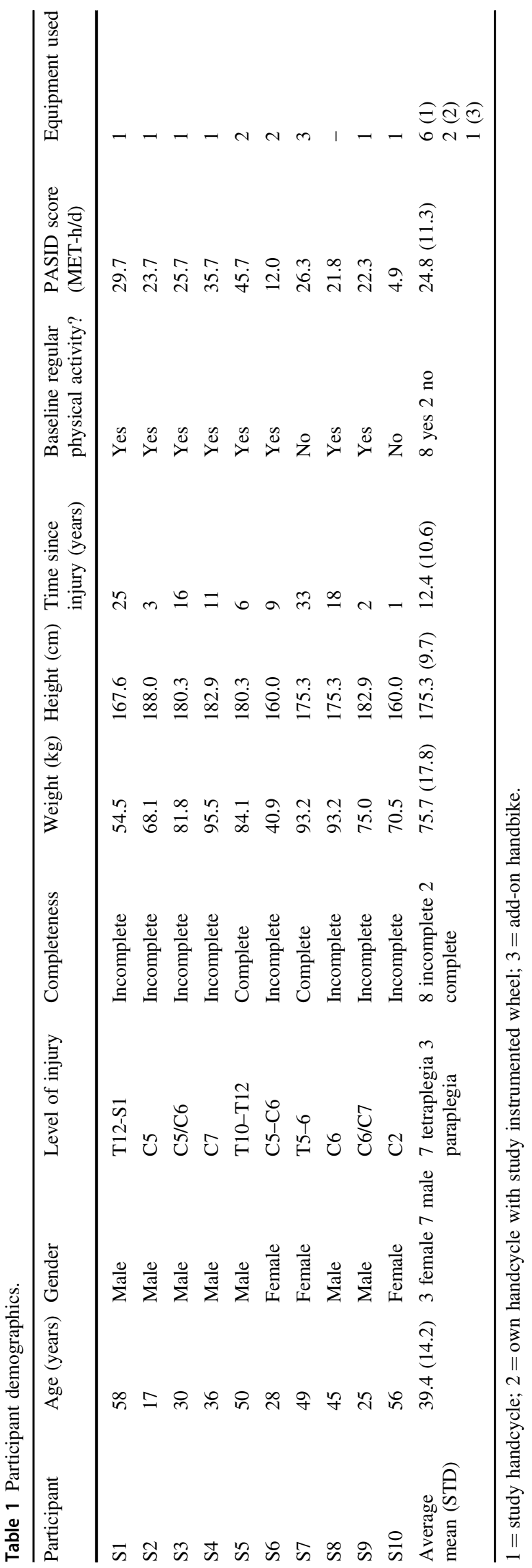

$(\mathrm{SD})=5.9$ (1.3) while the highest scored item was "I enjoy it" $(6.9(0.3))$.

Participants who completed at least one HIIT session $(n=9)$ completed the study-specific survey. All of them expressed a high level of satisfaction with the HIIT exercise $(100 \%$ very satisfied), working out at home $(89.9 \%$ very satisfied) and with a trainer $(100 \%$ very satisfied). Participants expressed mixed feelings about the difficulty of the exercise (Fig. 3). A majority felt their endurance, health, wheelchair propulsion, and transfer ability improved after the training period (Fig. 3). All expressed an interest in continuing HIIT and $100 \%$ would recommend this form of exercise to their peers.

\section{Preliminary efficacy and safety}

There were no study related adverse events. Fitness variables were examined for the 6 participants who completed at least 12 training sessions during the 6 weeks (Table 4). While some individuals did show improvement in fitness, no group level changes were found.

\section{Discussion}

This study sought to determine the feasibility of performing low-volume handcycling HIIT for 6 weeks. Several methods were used to facilitate acceptance including homebased workouts, a personal trainer, and a stationary handcycle setup. The results of the study showed that the handcycling HIIT program was feasible, safe and that a majority of the participants met the target power intensities during the workouts. The participants also found HIIT to be challenging but enjoyable and acceptable. They also perceived gains in endurance, function, and overall health however the objective pre-post fitness metrics showed mixed results regarding efficacy among individual participants.

\section{Feasibility of recruitment/retention}

There was high interest in the HIIT study from persons with tetraplegia. This may be due to the very limited options available for exercise in this subgroup. Thirty percent withdrew at various points in the study for reasons that were unrelated to the study. This is consistent with other longitudinal exercise studies involving persons with SCI $[24,25]$. The two participants with the lowest PASID scores, 12.0 (S6) and 4.9 (S10) both withdrew from the study for unrelated health issues. While ideal to target minimally active individuals for these types of studies retention of these individuals can also be very challenging. 
Table 2 Adherence with number of training sessions and HIIT criteria. Also shown are those participants who withdrew and participants who completed the pre-post testing measures.

\begin{tabular}{|c|c|c|c|c|c|c|c|c|}
\hline Participant ID & $\begin{array}{l}\text { Training } \\
\text { sessions } \\
\text { completed } \\
\text { count }(\%)\end{array}$ & $\begin{array}{l}\text { Sessions } \\
\text { meeting } \\
\text { power criteria } \\
\text { count }(\%)\end{array}$ & $\begin{array}{l}\text { Sessions } \\
\text { meeting HR } \\
\text { criteria } \\
\text { count }(\%)\end{array}$ & $\begin{array}{l}\text { Sessions } \\
\text { meeting RPE } \\
\text { criteria } \\
\text { count }(\%)\end{array}$ & $\begin{array}{l}\text { Sessions } \\
\text { meeting at } \\
\text { least one HIIT } \\
\text { criteria } \\
\text { count }(\%)\end{array}$ & $\begin{array}{l}\text { Sessions } \\
\text { meeting at } \\
\text { least two HIIT } \\
\text { criteria } \\
\text { count }(\%)\end{array}$ & $\begin{array}{l}\text { Sessions } \\
\text { meeting all } \\
\text { three HIIT } \\
\text { criteria } \\
\text { count }(\%)\end{array}$ & $\begin{array}{l}\text { Both pre-post } \\
\text { testing } \\
\text { completed? } \\
\text { (yes/no) }\end{array}$ \\
\hline S1 & $12(100)$ & $10(83.3)$ & 2 (16.7) & $1(8.3)$ & 11 (91.7) & 2 (16.7) & $0(0)$ & Yes \\
\hline S2 & $15(100)^{\mathrm{a}}$ & $3(20.0)$ & $1(6.7)$ & $2(13.3)$ & $5(33.3)$ & $1(6.7)$ & $0(0)$ & Yes \\
\hline S3 & $13(100)^{\mathrm{b}}$ & $13(100)$ & 11 (84.6) & $10(76.9)$ & $13(100)$ & $12(92.3)$ & $9(69.2)$ & Yes \\
\hline S4 & $6(50)$ & $6(100)$ & $4(66.7)$ & $0(0)$ & $6(100)$ & $4(66.7)$ & $0(0)$ & Yes \\
\hline S5 & $12(100)$ & $9(75)$ & $9(75)$ & $8(66.7)$ & $11(91.7)$ & $10(83.3)$ & $5(41.7)$ & Yes \\
\hline S6-W & $6(50)$ & $5(83.3)$ & $2(33.3)$ & $6(100)$ & $6(100)$ & $5(83.3)$ & $2(33.3)$ & No \\
\hline S7 & $12(100)$ & $5(41.7)$ & $7(58.3)$ & $9(75)$ & 11 (91.7) & $9(75)$ & $1(8.3)$ & Yes \\
\hline S8-W & $0(100)$ & N/A & N/A & N/A & N/A & N/A & N/A & No \\
\hline S9 & 12 (100) & $12(100)$ & $4(33.3)$ & $10(83.3)$ & $12(100)$ & $11(91.7)$ & $3(25)$ & Yes \\
\hline $\mathrm{S} 10-\mathrm{W}$ & $1(8.3)$ & $1(100)$ & $1(100)$ & $1(100)$ & $1(100)$ & $1(100)$ & $1(100)$ & No \\
\hline Total & 89 & 64 (71.9) & $41(46.1)$ & $47(52.8)$ & $76(85.4)$ & $55(61.8)$ & 21 (23.6) & 7 yes $/ 3$ no \\
\hline
\end{tabular}

$\mathrm{W}=$ participant withdrew for reasons unrelated to the study, $\%=$ percentage.

${ }^{\text {a }}$ Performed three extra sessions without trainer.

${ }^{b}$ Performed one extra supervised session.

Table 3 PACES group mean and standard deviation (SD) item and total scores $(n=9)$.

\begin{tabular}{lr}
\hline Items & Mean (SD) \\
\hline "I enjoy it" & $6.9(0.3)$ \\
"I feel interested" & $6.7(0.5)$ \\
"I like it" & $6.8(0.7)$ \\
"I find it pleasurable" & $6.0(1.7)$ \\
"I am very absorbed in this activity" & $6.1(1.3)$ \\
"It's a lot of fun" & $6.3(0.7)$ \\
"I find it energizing" & $5.9(1.3)$ \\
"It makes me happy" & $6.7(0.7)$ \\
"It's very pleasant" & $6.1(1.3)$ \\
"I feel good physically while doing it" & $6.6(1.3)$ \\
"It's very invigorating" & $6.3(0.9)$ \\
"I am not at all frustrated by it" & $6.4(0.5)$ \\
"It's very gratifying" & $6.5(0.5)$ \\
"It's very exhilarating" & $6.3(0.7)$ \\
"It's very stimulating" & $6.1(1.6)$ \\
"It gives me a strong sense of accomplishment" & $6.6(0.5)$ \\
"It's very refreshing" & $6.4(0.7)$ \\
"I felt as though there was nothing else, I would rather & $6.0(0.7)$ \\
be doing" & \\
Summative score (range 18-126) & $114.8(11.3)$ \\
\hline
\end{tabular}

\section{Adherence to weekly sessions and HIIT targets}

Adherence to the twice weekly sessions was excellent. All the participants were encouraged to do an extra workout per week on their own however only one person did. The main reasons given included the inability to independently transfer into the handcycle and lack of time. Handcycle transfers are very challenging and few individuals with SCI can do them independently. One person selected the add-on handcycle option just to avoid the transfer as she had wrist pain with transfers. Despite the issues with the transfer all participants enjoyed using either the handcycle or add-on handbike for the training. The lack of time for exercise issue is a common barrier among persons with SCI [3] and exercising twice a week in accordance with current guidelines (20 min at moderate intensity) is not enough to substantially alter cardiovascular and cardiometabolic health $[10,26]$. Future studies are needed to determine if two times a week of performing continuous vigorous activity or HIIT could make a greater impact on health outcomes.

Most participants were able to meet one or more of the targets for confirming adherence with HIIT. There was a higher level of adherence with the power criteria than the HR and RPE criteria not surprisingly as it was used to prescribe the exercise intensity. However S2 had multiple issues meeting the power targets due to difficulty with maintaining adequate hand grip, weak triceps control and lower leg spasticity during the sessions. With S7 there was decision made by the trainer to up the power target range midway into the training and while she fell short of the power targets she met criteria related to HR and RPE for these sessions.

The HR criteria was not met as often as power and could be related to the unreliability associated with HR measurement during short intervals, poor connection issues with the 


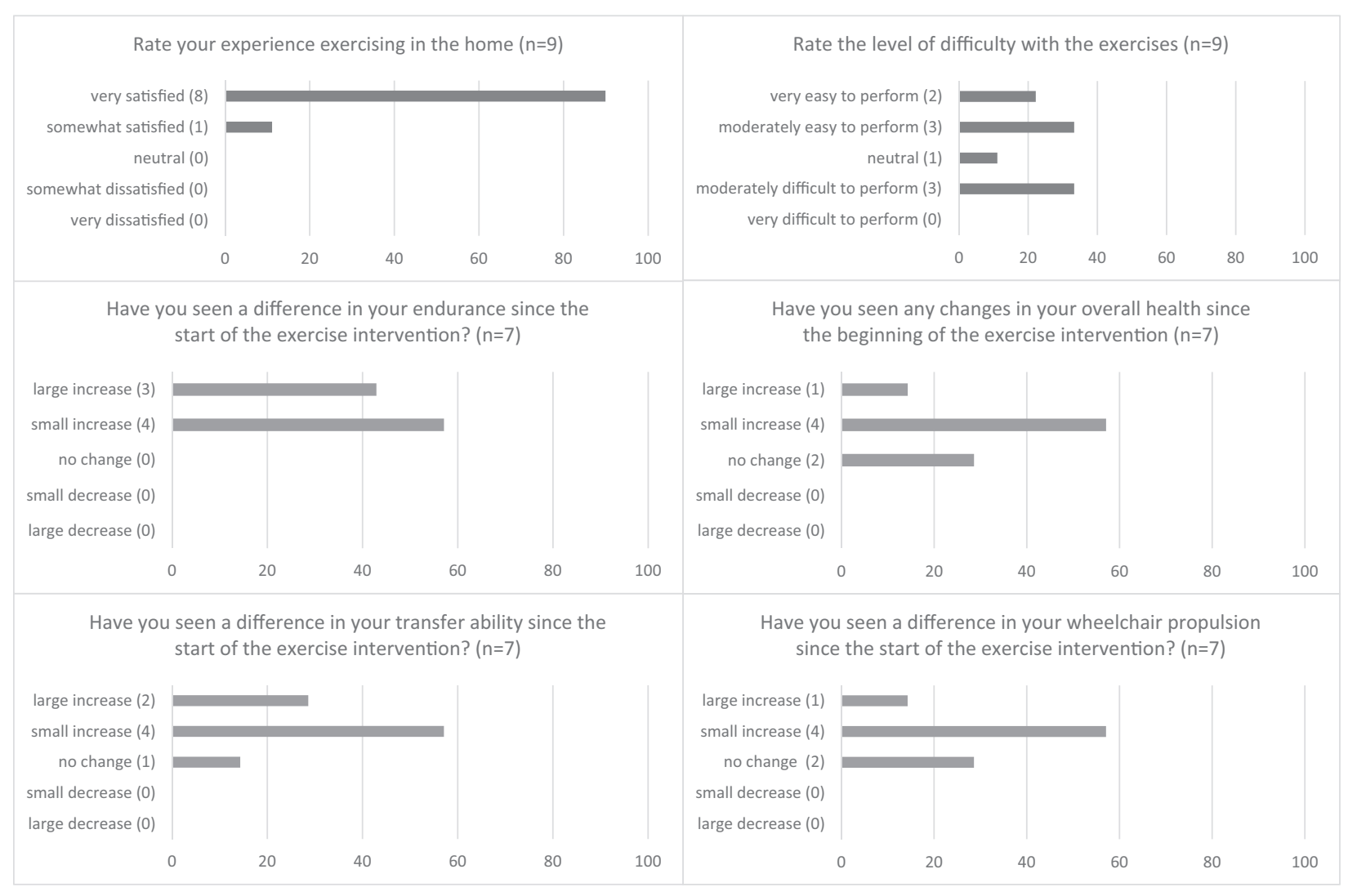

Fig. 3 Exit survey questions. Numbers in parentheses after responses are participant counts and bars represent the percentage of participants.

Table 4 Baseline, postintervention, and estimates of change in the fitness variables for participants who completed at least 12 sessions in 6 weeks $(n=6)$.

\begin{tabular}{lllll}
\hline Variable & Baseline mean (SD) & Postintervention mean (SD) & Change mean (SD) & $P$ value for change \\
\hline Peak $\mathrm{VO}_{2}(\mathrm{ml} / \mathrm{kg} / \mathrm{min})$ & $14.3(5.0)$ & $14.3(4.8)$ & $0(1.8)$ & 0.931 \\
Peak $\mathrm{VO}_{2}(\mathrm{ml} / \mathrm{min})$ & $1092.8(482.7)$ & $1086.2(441.2)$ & $-6.7(157.2)$ & 0.921 \\
Time to exhaustion (min:sec) & $6: 17(2: 59)$ & $6: 49(3: 39)$ & $0: 33(0.53)$ & 0.194 \\
Peak power output (W) & $60.0(33.3)$ & $65.0(38.5)$ & $5.0(8.4)$ & 0.203 \\
\hline
\end{tabular}

$\mathrm{VO}_{2}$ Oxygen consumption, $\mathrm{SD}$ standard deviation, min minutes, sec seconds, $\mathrm{ml}$ milliliters, $\mathrm{kg}$ kilograms.

sensors, blunted HR responses, and high variability observed in HR in SCI [27]. As a result HR may not be ideal for targeting HIIT intensity for persons with SCI. While power zones provide accurate targets the instrumentation involved is not as straightforward as wearing a HR monitor. The instrumentation for recording and displaying power output $(\$ 800)$ combined with the costs of a handcycle and/or add-on handbike (\$3500) may prohibit some individuals from being able to afford the equipment. The RPE criteria was only met in about half of the sessions and may be related to its questionable validity for gauging vigorous upper arm exercise intensity in SCI [28]. However RPE would be an ideal method for prescribing exercise intensity as it is more practical and inexpensive.

\section{Acceptability}

Both measures of acceptability were very positive. The PACES showed a high level of enjoyment on all the items. Without another form of exercise to compare to (e.g., continuous handcycling) it is unknown if the scores were due to the unique nature of HIIT or if continuous handcycling would have been enough to peak their enjoyment. However a study in SCI that compared HIIT arm ergometry to continuous moderate intensity arm ergometry found that people preferred HIIT [29]. Another study however found participants perceived higher risks with HIIT such as it may lead to or increase their shoulder pain [11]. Handcycling crank positioning was customized for each person and is very important 
for minimizing stress on the shoulders [14, 15]. As evidence is surfacing about the best locations to position the crank to optimize biomechanics and performance [30] it would be good for future studies to adopt standardized methods for ensuring proper fit and handcycling technique for HIIT.

This study found that some of the participants (see Supplementary files) came close to exceeding their physical capacity (HRMax) when performing HIIT however no one experienced any adverse study related health effects. Our participants were mostly with incomplete injuries and may have a less impaired sympathetic nervous system that is more capable of recovering from the extreme stress placed on the cardiovascular system. More studies are needed to investigate HIIT exercise applied to individuals with varying neurological levels and completeness of SCI to establish safety in drawing near to or exceeding physical capacity.

\section{Preliminary efficacy}

Studies on HIIT in SCI have shown mixed results so far with regards to efficacy $[11,31,32]$. Our study found that of the six participants who completed all the sessions three (S1, S2, and S9) showed an improvement, one (S5) had no change and two (S3 and S7) showed a decrease in peak $\mathrm{VO}_{2}$ postintervention. Participant S5 was highly active before the intervention (i.e., PASID = 45.7) therefore it is possible he was already at or near a peak level of fitness. Participant S3 was highly compliant with the HIIT protocol and reported large improvements (5 out of 5) in endurance, transfer ability, wheelchair propulsion and overall health after the intervention. Participant S7 showed a decrease in peak $\mathrm{VO}_{2}$ but a 20 $\mathrm{W}$ increase in PPO. For her it would appear that the training may have helped her get stronger but it did not increase her aerobic capacity. Like S3 she reported small improvements in endurance, wheelchair propulsion and overall health and a large improvement in transfer ability at the end of the intervention and good compliance with meeting HIIT criteria. It is possible that the differences in the mode of exercise (e.g., asynchronous arm ergometry vs. synchronous handcycling) could have impacted the test performance as the training used a different movement strategy and likely different muscle activation patterns than the exercise tests. It is also possible that the HIIT programming (1:1 min ratio) was not sufficient enough for eliciting the desired aerobic benefits. The 1:1 min ratio is a standard that has proven to be highly tolerable and effective in numerous other HIIT studies [5, 9, 33] however they all involved lower extremity HIIT workouts. Longer intervals may be more effective in upper body training due to the different oxygen uptake kinetics of upper body compared to lower body exercise [34]. Future research is needed to determine an optimal HIIT programming interval for handcycling HIIT for persons with SCI.

\section{Limitations}

Some of the participants felt they reached a muscular fatigue when performing HIIT before reaching an aerobic fatigue. Upper limb strength would be expected to increase over the course of doing HIIT however including targeted strengthening and stretching upper body exercises could condition the limbs for performing high workload activity and protect against developing overuse related pain and injuries. While the baseline laboratory fitness test provided guidance on where to set the HIIT power target intensities, when transitioning to the home we found the power targets needed to be adjusted for some individuals to obtain the desired response. This was likely due to the equipment, mode of exercise and body positioning setup for the $\max \mathrm{VO}_{2}$ test being different from the training sessions (e.g., asynchronous upright arm ergometry vs. synchronous recumbent handcycling). It would be beneficial in future studies to use a similar mode of cranking and body positioning during the exercise test and HIIT training.

\section{Conclusion}

Six weeks of handcycling HIIT appears to be a safe and feasible option for persons with paraplegia and tetraplegia. Working out with a trainer, the computer guided workout, and short duration training sessions may have attributed to the high adherence and positive feedback. Future work is needed to identify the optimal HIIT programming and number of weekly sessions that would be needed to elicit changes in cardiorespiratory and cardiometabolic outcomes.

Acknowledgements We would like to thank the participants with SCI who participated in this research. In addition, we express our gratitude to the following study coordinators and trainers Sarah Bass, Khara James, Anas Dighriri, and Ian Eckstein.

Funding The NIH React Center Pilot Studies Program at the University of Alabama, Birmingham, funded the study. Training equipment (handcycles, add-on handbike, and Garmin sensors and computer) was funded by an internal VA Rehabilitation R\&D Center Pilot Program Grant.

Author contributions AMK was responsible for designing the study protocol, analyzing data, interpreting the results, creating the tables and figures and writing the paper. CEG served as one of the study trainers, screened potentially eligible subjects, and assisted with data collection, data extraction and analysis. SJK provided oversight of the pre-post intervention exercise test data collections, assisted with data review and interpretation, paper review and editing. TMC assisted with protocol development, data collection, data interpretation, paper review and editing for content and readability. BCN assisted with interpreting results, reviewing and editing the paper for content and readability. 


\section{Compliance with ethical standards}

Conflict of interest The authors declare that they have no conflict of interest.

Ethical approval The study protocol was approved by the Institutional Review Board at the University of Pittsburgh (19040142) and was registered at Clinical Trials.gov (NCT03152110). We certify that all applicable institutional and governmental regulation concerning the ethical use of the human volunteers were followed during the course of this research.

Informed consent All participants received oral as well as written information about the study before written consent was obtained.

Publisher's note Springer Nature remains neutral with regard to jurisdictional claims in published maps and institutional affiliations.

\section{References}

1. Chen Y, Henson S, Jackson AB, Richards JS. Obesity intervention in persons with spinal cord injury. Spinal Cord. 2006;44:82-91.

2. Ginis KA, Latimer AE, Arbour-Nicitopoulos KP, Buchholz AC, Bray SR, Craven BC, et al. Leisure time physical activity in a population-based sample of people with spinal cord injury part I: demographic and injury-related correlates. Arch Phys Med Rehabilit. 2010;91:722-8.

3. Kehn M, Kroll T. Staying physically active after spinal cord injury: a qualitative exploration of barriers and facilitators to exercise participation. BMC Public Health. 2009;9:168.

4. Martin Ginis KA, van der Scheer JW, Latimer-Cheung AE, Barrow A, Bourne C, Carruthers $\mathrm{P}$, et al. Evidence-based scientific exercise guidelines for adults with spinal cord injury: an update and a new guideline. Spinal Cord. 2018;56:308-21.

5. Gibala MJ, Little JP, Macdonald MJ, Hawley JA. Physiological adaptations to low-volume, high-intensity interval training in health and disease. J Physiol. 2012;590:1077-84.

6. Jimenez-Maldonado A, Renteria I, Garcia-Suarez PC, MoncadaJimenez J, Freire-Royes LF. The impact of high-intensity interval training on brain derived neurotrophic factor in brain: a minireview. Front Neurosci. 2018;12:839.

7. Costa EC, Hay JL, Kehler DS, Boreskie KF, Arora RC, Umpierre $\mathrm{D}$, et al. Effects of high-intensity interval training versus moderateintensity continuous training on blood pressure in adults with pre- to established hypertension: a systematic review and meta-analysis of randomized trials. Sports Med. 2018;48:2127-42.

8. Schoenmakers P, Reed K, Van Der Woude L, Hettinga FJ. High intensity interval training in handcycling: the effects of a 7 week training intervention in able-bodied men. Front Physiol. 2016;7:638

9. Sawyer BJ, Tucker WJ, Bhammar DM, Ryder JR, Sweazea KL, Gaesser GA. Effects of high-intensity interval training and moderate-intensity continuous training on endothelial function and cardiometabolic risk markers in obese adults. J Appl Physiol. 2016;121:279-88.

10. Nightingale TE, Metcalfe RS, Vollaard NB, Bilzon JL. Exercise guidelines to promote cardiometabolic health in spinal cord injured humans: time to raise the intensity? Arch Phys Med Rehabilit. 2017;98:1693-704.

11. Gauthier C, Brosseau R, Hicks AL, Gagnon DH. Feasibility, safety, and preliminary effectiveness of a home-based selfmanaged high-intensity interval training program offered to long-term manual wheelchair users. Rehabilit Res Pract. 2018; 2018:8209360

12. Boninger ML, Cooper RA, Baldwin MA, Shimada SD, Koontz A. Wheelchair pushrim kinetics: body weight and median nerve function. Arch Phys Med Rehabilit. 1999;80:910-5.

13. Collinger JL, Impink BG, Ozawa H, Boninger ML. Effect of an intense wheelchair propulsion task on quantitative ultrasound of shoulder tendons. PM R. 2010;2:920-5.

14. Dallmeijer AJ, Zentgraaff ID, Zijp NI, van der Woude LH. Submaximal physical strain and peak performance in handcycling versus handrim wheelchair propulsion. Spinal Cord. 2004;42:91-8.

15. Arnet U, van Drongelen S, Scheel-Sailer A, van der Woude LH, Veeger DH. Shoulder load during synchronous handcycling and handrim wheelchair propulsion in persons with paraplegia. J Rehabilit Med. 2012;44:222-8.

16. Pinto N, Salassi JW 3rd, Donlin A, Schroeder J, Rozenek R. Effects of a 6-week upper extremity low-volume, high-intensity interval training program on oxygen uptake, peak power output, and total exercise time. J Strength Cond Res. 2019;33:1295-304.

17. Washburn RA, Zhu W, McAuley E, Frogley M, Figoni SF. The physical activity scale for individuals with physical disabilities: development and evaluation. Arch Phys Med Rehabilit. 2002;83: 193-200.

18. Maher JL, Cowan RE. Comparison of 1- versus 3-minute stage duration during arm ergometry in individuals with spinal cord injury. Arch Phys Med Rehabilit. 2016;97:1895-1900.

19. Maier T, Schmid L, Muller B, Steiner T, Wehrlin JP. Accuracy of cycling power meters against a mathematical model of treadmill cycling. Int J Sports Med. 2017;38:456-61.

20. Bouillod A, Pinot J, Soto-Romero G, Bertucci W, Grappe F. Validity, sensitivity, reproducibility, and robustness of the Powertap, stages, and garmin vector power meters in comparison with the SRM device. Int J Sports Physiol Perform. 2017;12:1023-30.

21. Taylor JL, Holland DJ, Spathis JG, Beetham KS, Wisloff U, Keating SE, et al. Guidelines for the delivery and monitoring of high intensity interval training in clinical populations. Prog Cardiovasc Dis. 2019;62:140-6.

22. Kendzierski D, DeCarlo KJ. Physical activity enjoyment scale: two validation studies. J Sport Exerc Psychol. 1991;13:50-64.

23. Brurok B, Helgerud J, Karlsen T, Leivseth G, Hoff J. Effect of aerobic high-intensity hybrid training on stroke volume and peak oxygen consumption in men with spinal cord injury. Am J Phys Med Rehabilit. 2011;90:407-14.

24. Bakkum AJ, Paulson TA, Bishop NC, Goosey-Tolfrey VL, Stolwijk-Swuste JM, van Kuppevelt DJ, et al. Effects of hybrid cycle and handcycle exercise on cardiovascular disease risk factors in people with spinal cord injury: a randomized controlled trial. J Rehabilit Med. 2015;47:523-30.

25. Crane DA, Hoffman JM, Reyes MR. Benefits of an exercise wellness program after spinal cord injury. J Spinal Cord Med. 2017;40:154-8.

26. Totosy de Zepetnek JO, Pelletier CA, Hicks AL, MacDonald MJ. Following the physical activity guidelines for adults with spinal cord injury for 16 weeks does not improve vascular health: a randomized controlled Trial. Arch Phys Med Rehabilit. 2015;96:1566-75.

27. Partida E, Mironets E, Hou S, Tom VJ. Cardiovascular dysfunction following spinal cord injury. Neural Regener Res. 2016;11:189-94.

28. van der Scheer JW, Hutchinson MJ, Paulson T, Martin Ginis KA, Goosey-Tolfrey VL. Reliability and validity of subjective measures of aerobic intensity in adults with spinal cord injury: a systematic review. PM R. 2018;10:194-207.

29. Astorino TA, Thum JS. Interval training elicits higher enjoyment versus moderate exercise in persons with spinal cord injury. $\mathrm{J}$ Spinal Cord Med. 2018;41:77-84. 
30. Vegter RJK, Mason BS, Sporrel B, Stone B, van der Woude LHV, Goosey-Tolfrey VL. Crank fore-aft position alters the distribution of work over the push and pull phase during synchronous recumbent handcycling of able-bodied participants. PLoS One. 2019;14:e0220943.

31. Graham K, Yarar-Fisher C, Li J, McCully KM, Rimmer JH, Powell D, et al. Effects of high-intensity interval training versus moderate-intensity training on cardiometabolic health markers in individuals with spinal cord injury: a pilot study. Top Spinal Cord Inj Rehabilit. 2019;25:248-59.
32. Harnish CR, Daniels JA, Caruso D. Training response to highintensity interval training in a 42-year-old man with chronic spinal cord injury. J Spinal Cord Med. 2017;40:246-9.

33. Boyne P, Dunning K, Carl D, Gerson M, Khoury J, Rockwell B, et al. High-intensity interval training and moderate-intensity continuous training in ambulatory chronic stroke: feasibility study. PhysTher. 2016;96:1533-44.

34. Koppo K, Bouckaert J, Jones AM. Oxygen uptake kinetics during high-intensity arm and leg exercise. Respir Physiol Neurobiol. 2002;133:241-50. 